\title{
39. Current Evolution of Meteoroids
}

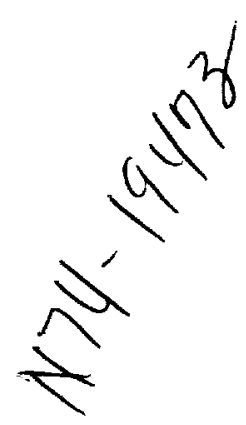

J. S. DohnanYI

Bellcomm, Inc.

Washington, D.C.

The observed mass distribution of meteoroids at 1 AU from the Sun is briefly reviewed in a survey that ranges over the bulk of the mass spectrum from micrometeoroids to meteorite parent objects. The evolution of meteoroids under the influence of collisions, planetary perturbations, the Poynting-Robertson effect and radiation pressure is then discussed.

Most micrometeoroids are expelled from the solar system by radiation pressure shortly after their production as secondary ejecta during impact by larger objects or as dust ejected by comets. Particles that survive will eventually be swept out by the PoyntingRobertson effect.

Meteoroids in the radio and photographic ranges are destroyed in collisions faster than they can be replaced by the production of secondary fragments during collisions between larger objects. The source of new particles needed to maintain the population of these meteoroids in a stationary distribution may be material expelled by comets.

The survival of large objects is limited by gravitational scattering during close planetary encounters and by collisions as well, if they spend sufficient time in the asteroid belt. The observed radiation-exposure ages of chondrites are shown to be consistent with this model.

THE Distribution of THE Masses of meteoroids $I_{\text {is governed by several processes (Whipple, }}$ 1967). Large numbers of new objects are injected into the solar system by comets. Many small objects are removed by the Poynting-Robertson effect (Robertson, 1937; Wyatt and Whipple, 1950); particles are destroyed by interparticle collisions and their shattering into fragments creates new particles (Whipple, 1967; Dohnanyi, 1967). The influence of these collisions on the distribution of meteoroid masses has recently been discussed by Dohnanyi (1970; to be referred to as D-I in this paper), who showed that the distribution of meteoroids in the photographic range and of fainter ones is not likely to be stationary unless many of the particles, destroyed by collisions, are replaced by new ones given off by comets; the influence of radiation pressure on the size distribution of such cometary debris was also stressed (D-I).

The orbital elements of meteoroids undergo frequent and random changes caused by planetary perturbations (Ópik, 1951; Arnold, 1965). The infuence of this process on the radiation exposure age distribution of meteorites has recently been discussed by Wetherill (1967) and Wetherill and Williams (1968). This age distribution was found to be sensitive to the survival times of metcorite-producing objects with respect to catastrophic collisions and to the rate at which these objects can "diffuse" through the solar system as a result of random gravitational perturbations causcd by close planetary encounters (Ópik, 1966). 
In this paper we shall discuss some aspects of the current evolution of the mass distribution of metcoroids; orbits will only be considered to the extent that they may influence the mass distribution. This will be shown to lead to a selfconsistent description of the dominating processes controlling the mass distribution of meteoroids with masses ranging from micrometeoroids to meteorite-producing objects having masses of tens of tons.

\section{OBSERVATIONAL EVIDENCE}

This section is a discussion of the observed distribution of sporadic meteoroids ranging in size from micrometeoroids to large objects.

The flux $n(m) d m$ of meteoroids having a mass in the range $m$ to $d m$ incident on a unit area per $2 \pi$ sr per unit time will be taken as:

$$
n(m) d m=a m^{-\alpha} d m
$$

where $a$ and $\alpha$ are constants in different mass ranges; $\alpha$ is known as the population index.

Figure 1 is a plot of the cumulative flux $N(m)$ of meteoroids into Earth's atmosphere per meter ${ }^{2}$ per sec per $2 \pi$ steradians having a mass of $m \mathrm{~kg}$ or greater.

$$
N(m)=\int_{m}^{M_{\infty}} n(M) d M
$$

where $M_{\infty}$ is the mass of the largest object included among meteoroids. Near the small mass limit of the distribution, I used the results of the Pioneer 8 and 9 data obtained by Berg and Gerloff (1970), multiplied by two to correct for the Earth's focusing effect. These authors have found an indication of a "cutoff" in the population of meteoroids, at a mass of about $5 \times 10^{-15} \mathrm{~kg}$, which sets the effective upper limit to the flux of penetrating particles, as seen in figure 1.

The points labelled Explorer XXIII and Pegasus are the influx rates measured by these satellites, and are based on calibrations by Naumann (1968) and Naumann et al. (1969). The penetration sensors aboard Explorer XXIII and Pegasus were calibrated in the laboratory by firing particles at meteoric velocities into sensors similar to those actually flown. Since many of these particles were accelerated gas dynamically, a fraction of their

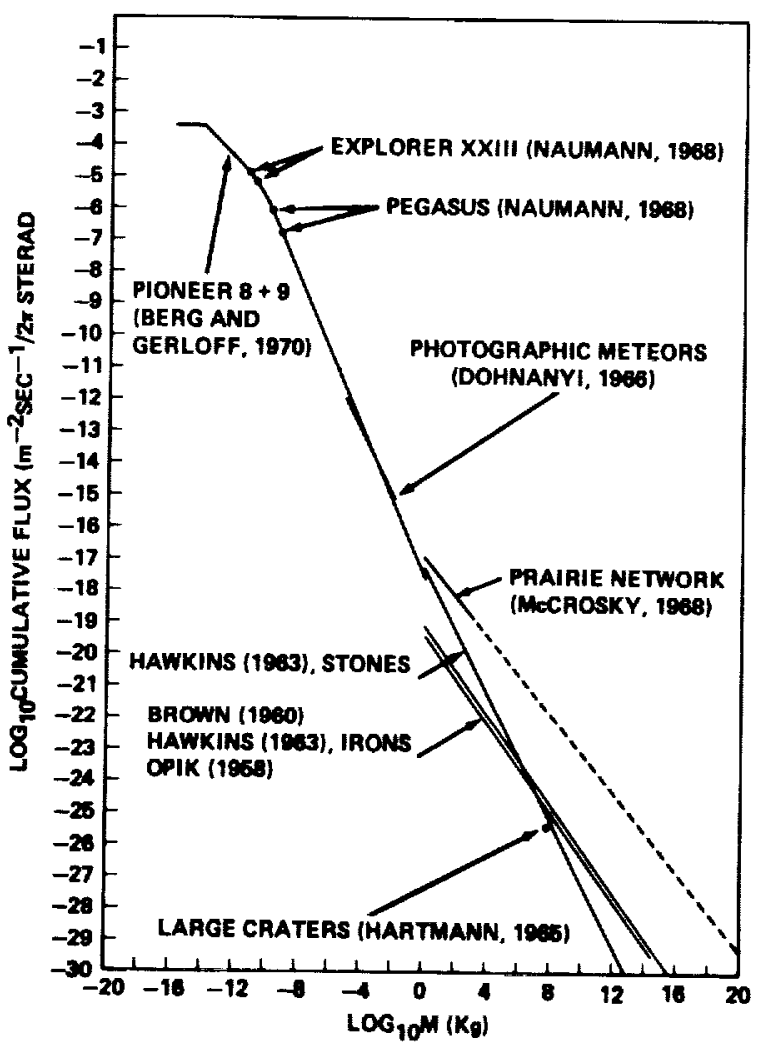

Figure 1.-Cumulative flux $\left(\mathrm{m}^{-2} \mathrm{~s}^{-1 / 2 \pi} \mathrm{sr}\right)$ of meteoroids into Earth's atmosphere having a mass of $m(\mathrm{~kg})$ or greater.

masses may have ablated during the acceleration, so that the indicated flux is likely an upper limit. Some micrometeoroids may, however, be fluffy and of low density (Soberman, 1971) and would be less penetrating than were the laboratory particles of equal mass, so that the indicated flux may also be a lower limit. The nominal flux is, however, in agreement with the penetration flux measured by the Ariel II satellite (Jennison et al., 1967). Since it is difficult to estimate precisely the uncertainties involved, an order of magnitude approximation may be the most accurate estimate that can be attained at the present time.

Data from visual, radar and photographic observations as well as zodiacal light studies have been considered before (Whipple, 1967; Dohnanyi, 1965). A best estimate to fit these data has been obtained in D-I; it has the form of equation (1) with

$\alpha=1.5$, for micrometeoroids, $m \leq 10^{-10} \mathrm{~kg}$ 
$\alpha=13 / 6$, for larger meteoroids but smaller than meteorite producing objects, $10^{-10} \mathrm{~kg} \leq m<1 \mathrm{~kg}$

This simple model gives a good fit to the data, as can be seen from the appropriate portions of the curve in figure 1. Numerical flux values are given in table 1.

Mass distributions obtained from satellite microphone measurements have been discussed by Kerridge (1970) and McDonnell (1971); because of calibration difficulties, many of these data are difficult to interpret. In those cases where calibration difficulties have likely been overcome, the results are comparable to the penetration data (Kerridge, 1970).

Mass fluxes of micrometeoroids estimated from particle collection experiments on board rockets and satellites are subject to uncertainties arising from contamination and identification difficulties (see Fechtig et al., 1968; Dohnanyi, 1971a, for an annotated bibliography) and will not be employed in this study. Many of these particles are comparable to or smaller than the wavelength of light; a discussion of the interaction of such small particles with the solar radiation ficld requires a discussion of interference effects and is beyond the scope of this paper.

The influx rate of metcorite-producing objects has been estimated variously by Brown (1960), Hawkins (1963), and Opik (1958); their estimates are plotted in figure 1. These data are based on the mass distribution of recovered meteorites and their estimated rates of fall. Hartmann's (1965) estimate of the flux rate of large crater-producing objects is also indicated.

Precise photographic observation of fireballs from the Prairie Network Project led McCrosky (1968b) to obtain as the cumulative flux (in MKS units) for these objects:

$$
N(m)=10^{-16.96} m^{-0.62} \quad 1 \leq m \leq 10^{4} \mathrm{~kg}
$$

As can be seen from figure 1, this flux is about an order of magnitude higher than that of Hawkins' (1963) stones and about two orders of magnitude higher than the other carlier estimates. Extrapolation (dashed line in fig. 1) of the Prairie Network data leads to an even greater difference. Uncertainties in the photometric masses of these objects are not believed to span this discrepancy
Table 1.-Differential Flux of Meteoroids $\mathrm{n}(\mathrm{m})=\mathrm{am}^{-\alpha}$ for Different Mass Ranges ${ }^{a}$

\begin{tabular}{lcr}
\hline \multicolumn{1}{c}{ Mass range } & $a, m^{-2} \mathrm{~s}^{-1} \mathrm{~kg}^{\alpha-1} / 2 \pi \mathrm{sr}$ & $\alpha$ \\
\hline$m<5 \times 10^{-15} \mathrm{~kg}$ & & $<3 / 2$ \\
$5 \times 10^{-16} \mathrm{~kg} \leq m \leq 10^{-10} \mathrm{~kg}$ & $1.4 \times 10^{-11}$ & $3 / 2$ \\
$10^{-10} \mathrm{~kg} \leq m<1 \mathrm{~kg}$ & $3 \times 10^{-18}$ & $13 / 6$
\end{tabular}

a See equation (1) in text.

(McCrosky and Ceplecha, 1970); low density, fragile objects whose fragments do not survive atmospheric entry are believed responsible for the higher flux of fireballs than had been estimated earlicr for meteorite producing objects.

\section{INFLUENCE OF COLLISIONS}

Meteoroids frequently undergo mutual collisions. Since these collisions are inelastic, the target particles may either lose a small portion of their mass (erosive collisions) or be completely broken up (catastrophic collisions). The net result is a change in the meteoroid distribution.

The equation that expresses the dependence of the population on collisions can be written as

$$
\begin{aligned}
\frac{\partial f(m, t)}{\partial t} d m= & \left.\frac{\partial f(m, t)}{\partial t} d m\right|_{\text {erosion }} \\
& +\left.\frac{\partial f(m, t)}{\partial t} d m\right|_{\text {estastrophle collisions }} \\
& +\left.\frac{\partial f(m, t)}{\partial t} d m\right|_{\text {oreation by fragmentation }}
\end{aligned}
$$

where $f(m t) d m$ is the particle number density function, i.e., the number of particles per unit volume of space in the mass range $m$ to $m+d m$. The number density $f(m, t) d m$ is perpetually altered by erosive and catastrophic collisions and the creation of fragments in the mass range $m$ to $m+d m$ by the crushing of larger objects during inclastic collisions.

It has been shown (Dohnanyi, 1969 and D-I) that, for a distribution with a population index $\alpha=13 / 6$ the contribution of particle creation, expressed by the last term in equation (5), is 
minor compared with the other two processes. The influence of collisions on the distribution of small particles is then dominated by catastrophic collisions, while erosion dominates the distribution of large particles (D-I).

The influence of catastrophic collisions on the particle population is readily calculated. We take for the number density of small objects in the mass range $m$ to $m+d m$ at $1 \mathrm{AU}$ from the Sun

$$
f(m)=\frac{1}{2} \frac{4}{V_{\infty}} n(m)
$$

Here $V_{\infty}$ is the average Earth entry velocity and $n(m)$ is given in table 1 ; the factor $1 / 2$ is included to correct for the Earth's gravitational focusing effect, the factor 4 results from averaging the velocity distribution over all directions and $n(m)$ is given by table 1 .

Using earlier results from photographic meteors (Dohnanyi, 1966) we take $V_{\infty}=20.6 \mathrm{~km} / \mathrm{s}$ and an average collision velocity for sporadic meteoroids equal to their mean geocentric velocity of 17.3 $\mathrm{km} / \mathrm{s}$. In D-I, the largest metcoroid mass, $\Gamma^{\prime}$, that is catastrophically disrupted by impact of a projectile meteoroid of unit mass, was estimated (Moore and Gault, 1965; Moore and Robertson, 1966). The result is $\Gamma^{\prime} \approx 7.5 \times 10^{4}$ for basalt particles and about an order of magnitude smaller for pumice particles.

Assuming a steady state distribution, one can readily calculate how many particles in this environment will survive catastrophic collisions after a time $t$. The result is shown in figure 2, which is a plot, for basalt-like particles, of the number density of particles that survive disruption after various time intervals, as indicated.

It is readily seen, from figure 2 , that the heaviest toll is taken from particles in the faint radiometeor range: less than 0.1 percent of these particles survive disruptive collisions in the mass range $10^{-9} \mathrm{~kg}<m<10^{-5} \mathrm{~kg}$ during a time interval of about $10^{5} \mathrm{yr}$. Larger particles survive longer because the number of projectile masses lethal to larger objects decreases; micrometeoroids, on the other hand, have a longer survival time for disruptive collisions because the distribution of small objects that disrupt them tapers off.

The true number of surviving micrometeoroids is smaller than indicated in figure 2, since the Poynting-Robertson effect will cause many of

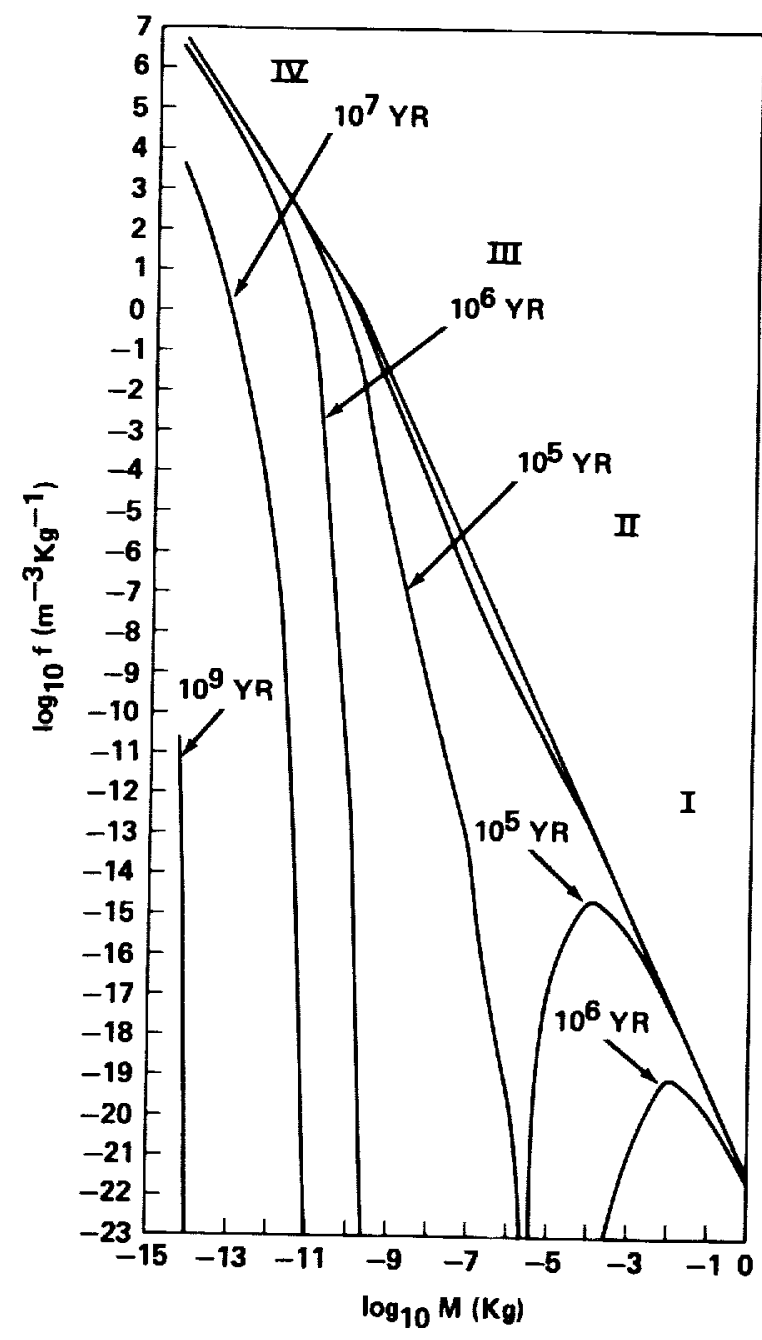

Figure 2.-Number density, $f(m, t) d m$, per meter ${ }^{3}$ of meteoroids having masses in the range $m$ to $m+d m$ $(\mathrm{kg})$ is plotted for different times $t$, as indicated, for particles surviving disruptive collisions in a stationary population $f(m, o)$.

these particles to spiral in toward the Sun. This is indicated as a function of particle mass in figure 3 , which is a plot of metcoroid survival times with respect to removal and destruction processes.

The survival time with respect to catastrophic collisions is made up of two main contributions:

where

$$
1 / \tau=1 / \tau_{c c}+1 / \tau_{s}
$$

$$
1 / \tau_{c c}(m)=k m^{2 / 3} \int_{m / \mathbf{\Gamma}^{\prime}}^{M_{\infty}} f(M) d \boldsymbol{M}
$$

and 


$$
1 / \tau_{\star}(m)=k \int_{m / \Gamma^{\prime}}^{M_{\infty}} M^{2 / 3 f}(M) d M
$$

$\tau_{c c}(m)$ is the survival time of a particle with respect to fragmentation by a comparatively small projectile particle, and $\tau_{s}(m)$ is the survival time of a particle with respect to being swept up by larger objects. The quantity $K$ is defined in (D-I) as

$$
K=\left(3 \pi^{1 / 2} / 4 \rho\right)^{2 / 3}\langle V\rangle
$$

where $\rho$ is the material density of the particles and $\langle V\rangle$ is the average encounter speed.

Figure 3 also shows a plot of the particle lifetime $\tau_{P R}$ with respect to the Poynting-Robertson effect for a particle of material density of $3.5 \mathrm{~g} \mathrm{~cm}^{-3}$ like that of basalt, and a material density of $1 / 3 \mathrm{~g} \mathrm{~cm}^{-3}$ which resembles pumice.

The time for a particle to erode to $1 / 2$ of its radius $\tau_{E}$ is plotted for a metcoroid with a density $3.5 \mathrm{~g} \mathrm{~cm}^{-3}$ resembling basalt in composition for two linear erosion rates: $100 \AA \mathrm{yr}^{-1}$, which is an upper limit obtained carlier by Whipple (1967), and $1 \AA \mathrm{yr}^{-1}$, a recent estimate based on cosmic ray track densities in glass removed from Surveyor 3 spacecraft, as well as in some lunar samples (Fleischer et al., 1971).
The line labelled "gravitational lifetime" is the effective survival time of a particle in an Earthcrossing orbit with respect to being swept up by the Earth (Öpik, 1966). The actual time a particle is expected to spend in Earth-crossing orbit is considerably shorter (Wetherill, 1968); multiple near encounters give rise to a random walk process causing the particle to "diffuse" out of the region where its motion may be perturbed by the gravitational field of the Earth.

Comparison of survival times with respect to the various dynamic processes plotted in figure 3 indicates that the Poynting-Robertson effect determines the survival times for micrometeoroids. Also, the survival times for disruptive collisions of micrometeoroids with masses $m<10^{-12} \mathrm{~kg}$ is dominated by the swceping-up action of larger particles. Erosion is negligible for small objects. Catastrophic collisions with relatively small projectile particles dominate the survival times of particles in a mass range of $10^{-10} \mathrm{~kg}<m<10^{3} \mathrm{~kg}$; larger objects will be dispersed by random walk from the vicinity of Earth's orbit long before they may be destroyed there by collisions.

Survival times with respect to cetastrophic collisions of asteroids in the asteroid belt

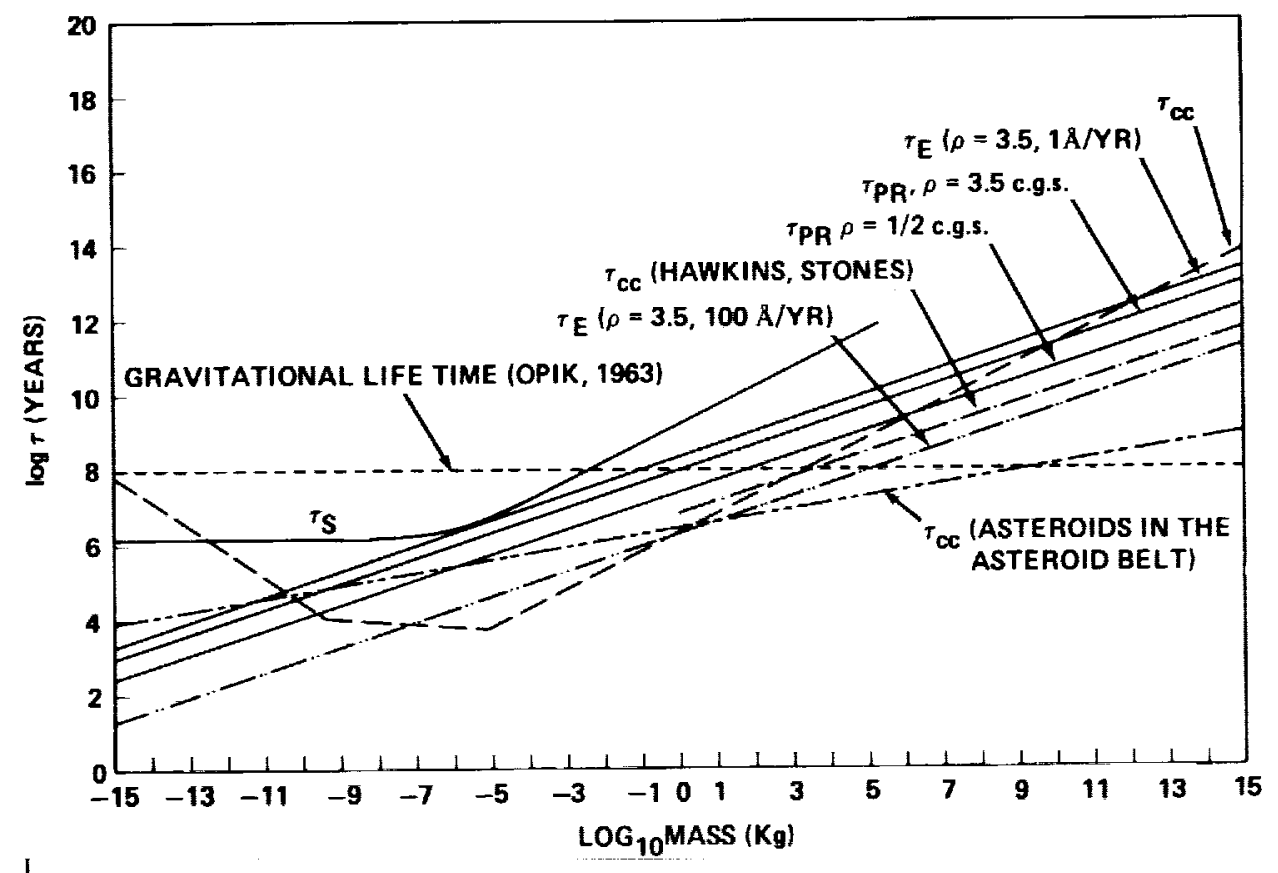

Frgure 3-Survival times as a function of mass of stray objects with respect to different loss processes, as indicated. 
(Dohnanyi, 1969) and the extrapolated values of these survival times for small masses are indicated in figure 3 for the sake of comparison.

\section{INFLUENCE OF RADIATION PRESSURE ON THE DISTRIBUTION OF METEOROID MASSES}

A certain amount of radiation pressure, in a direction away from the Sun, is exerted by the Sun's light on all objects in the solar system (Robertson, 1937; van de Hulst, 1962). The rcsult is a decrease, with decreasing particle radius, in the central force attracting the particle to the Sun. For particle radii smaller than a certain critical value, the electromagnetic force exceeds the gravitational force and the particle is blown out of the solar system. This critical radius is a function of the material density of the particle and its optical properties.

Consequently, the size distributions of particles injected by a comet into orbits about the Sun with an initial angular momentum equal to that of the comot will have a "cutoff" below a certain critical size due to radiation pressure. This problem was discussed in D-I (also Harwit, 1963), and the results are summarized in figure 4. This figure is a plot of the critical particle mass just blown away by radiation pressure when ejected at the perihelion of some parent comet having an eccentricity $e$, as indicated. The particles are assumed opaque and particle material densitics of $1 / 2,1,2$, and $3.5 \times 10^{3} \mathrm{~kg} / \mathrm{m}^{3}$ have been considered. The eccentricities of some major showers are also indicated. One may assume that each comet that presumably gave rise to a major shower had, at the time it created the shower, an eccentricity similar to that of the shower. The intersection of each horizontal line, representing the eccentricity of the shower, with any line labelled by a density gives the smallest particle mass with the given density that can be present in the shower, according to geometric optics.

It is readily seen, from figure 4, that none of the major showers would initially contain particles smaller than about $10^{-9}$ to $10^{-12} \mathrm{~kg}$ having a density of $1 / 2 \times 10^{3} \mathrm{~kg} / \mathrm{m}^{3}$. The radiation pressure cutoff on the shower masses occurs in the range of $10^{-11}$ to $10^{-13} \mathrm{~kg}$ for meteoroids having a density similar to basalt $\left(3.5 \times 10^{3} \mathrm{~kg} / \mathrm{m}^{3}\right)$. For particles having a mass of about $10^{-15} \mathrm{~kg}$ or smaller, interference effects come into play and a discussion of

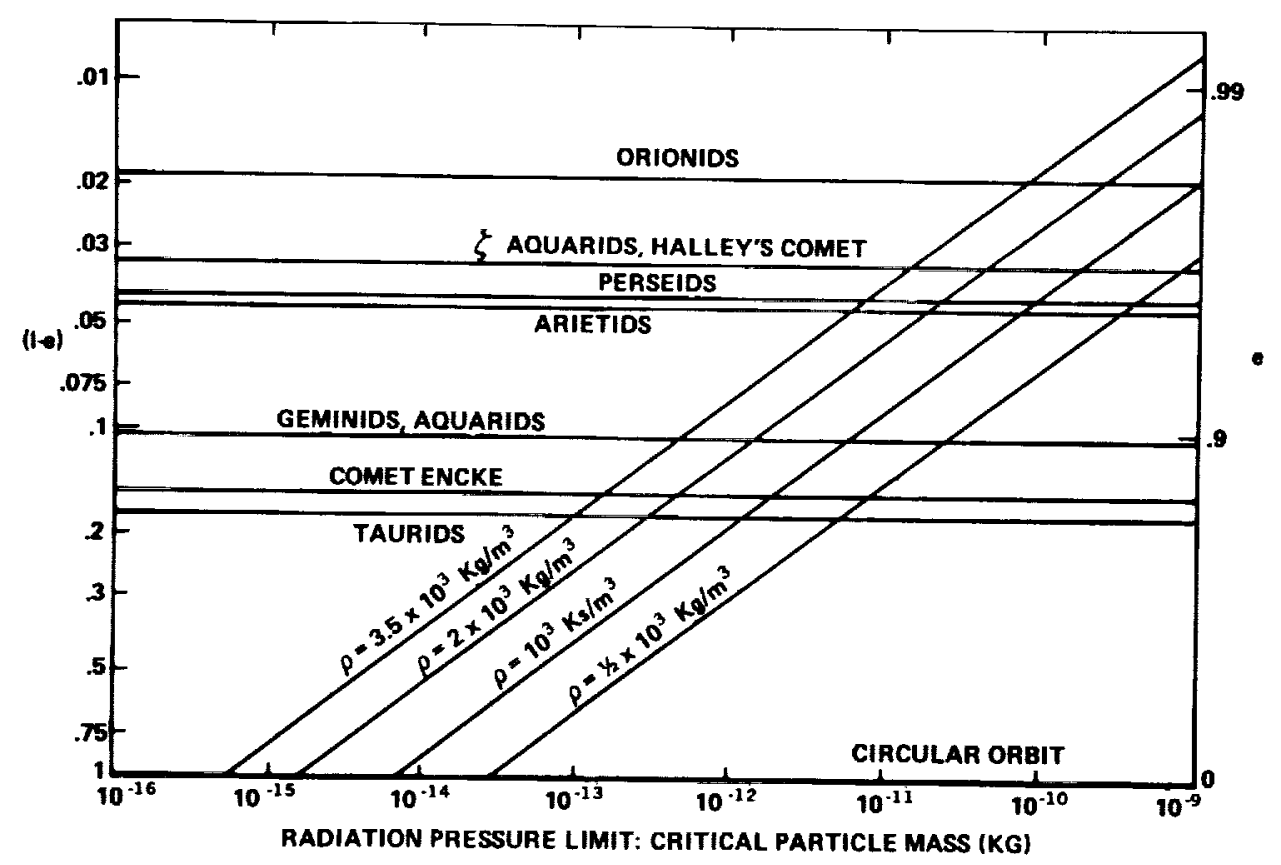

Figure 4.- Radiation pressure limit for particles of different material densities $\rho$ ejected by some parent object at perihelion; $e$ is the eccentrieity of the parent object. 
the stability of those very small particles is beyond the scope of this paper.

Similar considerations apply to fragments ejected from a parent body after an inelastic collision with another, smaller, meteoroid. In order to see, in detail, the effect under discussion, wi consider the eccentricity $E$ and semimajor axis $A$ of a fragment ejected from a parent body with eccentricity $e$ and semimajor axis $a$. Following D-I we denote by $\nu$ the ratio of the total eentral force on the fragment particle, i.e., the force of gravity reduced by radiation pressure, to the gravitational force acting on it. Since typical ejection velocities are small compared with the velocity of parent objects (Gault et al., 1963; Dohnanyi, 1971b) we take the heliocentric velocity of the ejecta equal to that of the parent objects immediately after impact. Assuming conservation of angular momentum, it can then be shown that

$$
A=\nu a /[1-2 a(1-\nu) / r]
$$

where $r$ is the radial distance from the Sun to the point of collision. Since $\nu \leq 1$, by definition, it can be shown from equation (11) that the semimajor axis, $A$, of the ejecta is always greater than the semimajor axis of the parent object.

It is readily seen from equation (11) that if

$$
a / r \geq 2 /(1-\nu)
$$

the ejecta will be expelled from the solar system by radiation pressure.

According to equation (12), elimination from the solar system by radiation pressure is favored for collisions near perihelion (i.e., small $a / r$ ) and since the particle number density as well as the encounter velocity increases rapidly toward the Sun (D-I), we may expect most collisions to occur near the perihelion of the colliding objects and hence figure 4 may also be applied to collisions, as a rough approximation. ${ }^{*}$ It may then be concluded that the contribution to the micrometeoroid population of fragments created during collisions by larger objects is much reduced by the elimination of these ejecta by radiation pressure.

- An exception occurs for objects having a node through the asteroid belt; depending on their orbital elements, it may then be physically possible for such objects to experience a more severe collisional environment in the asteroid belt than at perihelion.
The smaller the density of the fragment, the stronger is the effect of radiation pressure under discussion; this would seem to create a natural selection favoring the elimination of fluffy particles.

It has been shown in D-I that a population of objects with a mass distribution having a population index of $13 / 6$ is not stable; because of collisions, particles in any mass range are destroyed faster than they can be replaced by the creation of fragments of the same mass range. Hence, if the present distribution of meteoroids has reached an approximately steady state configuration, it is necessary to have a source of meteoroids replenishing the particles destroyed by collisions but not replaced by fragments. If meteor showers are indeed the required source which replenishes the population of sporadic meteoroids, and if the known major showers and comets are representative of the source of meteoroids, one would expect a drop in the population of sporadic meteoroids with masses smaller than $10^{-9}$ to $10^{-11} \mathrm{~kg}$, depending on their density. This conclusion appears to be borne out by observation, as is indicated in the changing slope of the sporadic meteoroid mass spectrum around $10^{-10} \mathrm{~kg}$.

\section{RADIATION AGES OF METEORITES}

The survival time of large meteoroids with respect to catastrophic collisions increases monotonically with mass (fig. 3); the survival time of sufficiently large objects, therefore, will be dominated by the dispersive effects of gravitational perturbations. Taking the mean dispersal time of an object $\tau_{d}$ to be shorter than $10^{2}$ million years, which is the lifetime of objects with respect to being swept up by the Earth, we see from figure 3 that the survival time of objects with masses of hundreds of $\mathrm{kg}$ will be limited by $\tau_{d}$. This, however, does not necessarily hold for objects whose orbits cross the asteroid belt; depending on the relative time spent in the asteroid belt as well as on the average encounter velocity with asteroids, the survival time of these objects may still be collision dominated.

Figure 5 is a plot of the observed rate of fall of chondritic meteorites, as given by Wetherill (1969) in different cosmic ray exposure age ranges. It can be seen, in figure 5 , that few objects have 


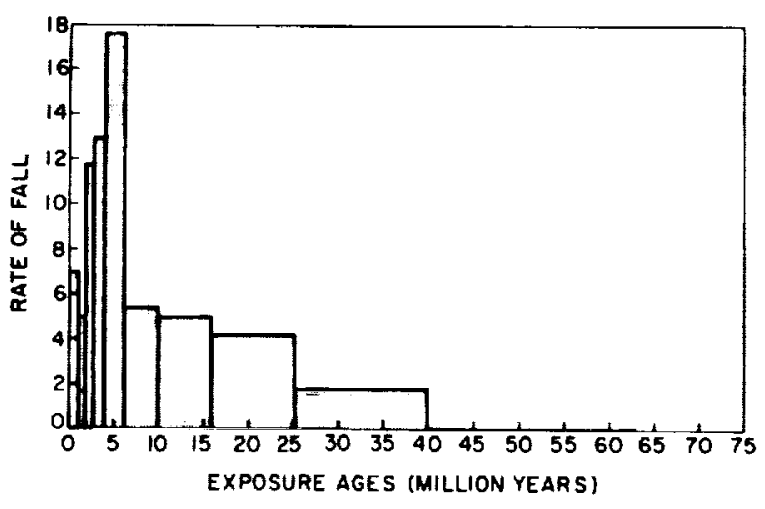

Figdre 5.-Observed cosmic-ray exposure ages of chondritic meteorites (Wetherill, 1969).

ages smaller than a million years. Under steady state conditions it appears, therefore, that most of these objects originated in a region of the solar system far away from Farth's orbit, and spent some time on their way to the Earth's vicinity. Consequently, very few newly created meteorites are present.

We therefore consider a simplified model for metcorite producing objects. We shall assume that the meteorite producing objects have orbits (cf., Wetherill and Williams, 1968; Wetherill, 1969; and Anders, 1971, for a more general discussion) with nodes that do not intersect Earth's orbit, but do intersect the asteroid belt. This latter assumption is reasonable if we assume that the orbits of these objects resemble somewhat those of the Prairie Network fireballs (McCrosky, 1968a). Using typical orbital elements for these objects, we have

$$
\begin{aligned}
& q^{\prime} \sim 4 \mathrm{AU} \\
& e \sim 0.7 \\
& i \sim 10^{\circ}
\end{aligned}
$$

where $q$ is the aphelion distance, $e$ is the eccentricity of the orbit and $i$ is the inclination. It is readily shown that such an object spends about 75 percent of its time at a solar distance between $2.2 \mathrm{AU}$ and aphelion. If all this time is spent within the asteroid belt, then use of a stcady state asteroidal mass distribution (Dohnanyi, 1969) together with a mean encounter velocity with asteroids of about $11 \mathrm{~km} \mathrm{~s}^{-1}$ (as suggested by the orbital elements of equation (13)) leads, for an object with a mass of $10^{4} \mathrm{~kg}$, to

$$
\tau_{c c} \sim 1 \text { million years }
$$

where $\tau_{c c}$ is the survival time of the object with respect to catastrophic collisions with asteroids. We chose a mass of $10^{4} \mathrm{~kg}$ as a typical meteorite parent object; much smaller objects are likely to produce few, if any, meteorites after atmospheric entry and larger objects are comparatively so scarce that their contribution to the production of meteorites will be neglected here. The value of 1 million $\mathrm{yr}$ for $\tau_{c c}$ is an underestimate, since our test object is not likely to spend all of its time near the center of the asteroid belt; indeed, having an aphelion distance of $4 \mathrm{AU}$, it will spend about 40 percent of its time beyond $3.5 \mathrm{AU}$ from the Sun. Thus, taking $3.5 \mathrm{AU}$ as the outer bound of the asteroid belt,

$$
\tau_{c c} \sim 2 \cdot 10^{5} \mathrm{yr}
$$

appears a more reasonable estimate.

The cumulative number of $10^{4} \mathrm{~kg}$ objects, $h(T)$, having an age of $T$ million years or longer is then

$$
h(T)=h_{0} e^{-T / r_{c o}}
$$

where $h_{0}$ is the total number of our meteorite parent objects with masses of $10^{4} \mathrm{~kg}$. It is assumed, in equation (16), that these objects are in a steady state distribution and $h_{0}$ and $h(T)$ are thercfore independent of time.

We now assume that these objects are occasionally perturbed into Earth crossing orbits with node $(\mathrm{s})$ oscillating around $1 \mathrm{AU}$ so that collision with the Earth becomes possible and the objects may then be recovered as meteorites. In order for this to happen, one or both of the nodes must be at a distance of $1 \mathrm{AU}$ from the Sun which means that, for non-zero inclinations, the aphelion of the orbit will be some distance above the ecliptic and thus above the central region of the asteroid belt. The collisional survival time will be correspondingly lengthened for objects in such Earthcrossing orbits. As a first approximation, we assume, therefore, that these Earth-crossing objects will have survival times determined by the random gravitational scattering in close encounters with the Earth, i.e., the collisional survival times of these Earth-crossing orbits are relatively long. 
We may then write

$$
\frac{\partial n_{e}^{\prime}(t)}{\partial t} \approx-\frac{n_{e}^{\prime}(t)}{y}+\frac{h(t)}{x}
$$

where $n_{\theta}^{\prime}(t)$ is the number density per unit volume of 10-ton objects in Earth-crossing orbit at a time $t$, and $h(t)$ is the number density per unit volume of 10-ton objects in previous, non-Earth-crossing orbits. Quantity $1 / y$ is the a priori probability, per unit time, for any Earth-crossing object to be scattered out of Earth-crossing orbit by a close encounter with the Earth and $1 / x$ is the a priori probability per unit time for a non-Earthcrossing meteorite parent object to be scattered into an Earth-crossing orbit.

We assume steady state conditions:

$$
\partial n_{e}{ }^{\prime}(t) / \partial t=0
$$

and hence

$$
n_{e}^{\prime}=(y / x) h
$$

as one would expect.

To calculate the distribution of the ages $T$ of these objects, we replace $t$ by $T$ in Equation (17) and get

$$
\partial n_{e}(T) / \partial T=-n_{\varepsilon}(T) / y+h(T) / x
$$

where $\left[\partial n_{e}(T) / \partial T\right] d T$ is the number density per unit volume of 10-ton objects with ages in the range of $T$ to $T+d T$, and $n_{e}(T)$ and $h(T)$ are the number of Earth-crossing and non Earthcrossing objects, respectively, per unit volume with ages greater than $T$. Because of the imposition of steady state conditions, $n_{\varepsilon}$ and $h$ are given by equation (19), for $T=0$, i.e., the number of objects for all ages is given by equation (19). Using equations (16), (19) and (20) we get:

$\frac{\partial n_{e}(T)}{\partial T}=n_{e}(0)\left(e^{-T / \tau_{c c}}-e^{-T / y}\right) /\left(\tau_{c c}-y\right), \tau_{c c} \neq y$

and

$$
\frac{\partial n_{e}(T)}{\partial T}=n_{e}(0) T\left(e^{-T / y}\right) / y^{2}, \tau_{c c}=y
$$

The expression for $\left[\partial n_{e}(T) / \partial T\right] d T$ determined by equation (21) or (22), gives the number of meteorite producing objects having an age of $T$ to $T+d T$ and is therefore proportional to the number of meteorites in that cosmic ray exposureage range. It is readily seen, from equations (21) and (22), that the number of "young" meteorites having ages much smaller than $y$ and $\tau_{c c}$ is zero. This happens because a finite time is required for an object, which has just been created in a nonEarth-crossing orbit, to "find its way" to an Earth-crossing orbit.

A comparison of equation (21) with the observed cosmic ray exposure ages of chondritic meteorites based on a compilation by Wetherill (1969) is plotted in figure 6 . The data published by Wetherill (1969) appear in the form of histograms (fig. 5) representing the rates of fall for meteorites in various ranges of cosmic ray exposure ages. In figure 6, Wetherill's data are reduced to represent the differential distribution $\partial n_{e}(T) / \partial T$ of cosmic ray exposure ages per unit exposure-age range. Taking

$$
n_{e}(0)=68.95
$$

which is the total rate of fall for all exposure ages given by Wetherill (1969), $\partial n_{e}(T) / \partial T$ from equation (21) is then plotted in figure 6 for several combinations of the survival times $\tau_{c c}$ and $y$.

It can be seen from equations (21) and (22) that $\partial n_{e}(T) / \partial T$ is symmetric with respect to $\tau_{c c}$ and $y$. This means that if either $\tau_{e c}$ or $y$ is very short, there will be a peak in the distribution of meteorites with very short exposure ages. This happens because if $\tau_{c c}$ is short, young objects are favored and if $y$ is short, meteorites do not have enough time to "grow old" in Earth-crossing orbits. If, on the other hand, either $\tau_{c c}$ or $y$ is long, the distribution has a long exponential tail containing many old meteorites. This happens because relatively many objects escape catastrophic collisions if $\tau_{c c}$ is long, which results in a corresponding abundance of old objects. If $y$ is long, then meteorites in Earth crossing orbits stay around long enough for many of them to age there. If $\tau_{c c}$ is comparable to $y$, then most objects have an age of that same order of magnitude, i.e., a narrowly peaked distribution of radiation ages.

It can be seen, from figure 5 , that the best estimate of $2 \times 10^{6} \mathrm{yr}$ for $\tau_{c c}$ (cq. (15)) combined with a gravitational survival time in Earthcrossing orbit of about $2 \times 10^{6}$ yr provides a reasonable fit to the obscrvational data.

We now consider the frequency of metcorite falls. According to the results of the Prairie 


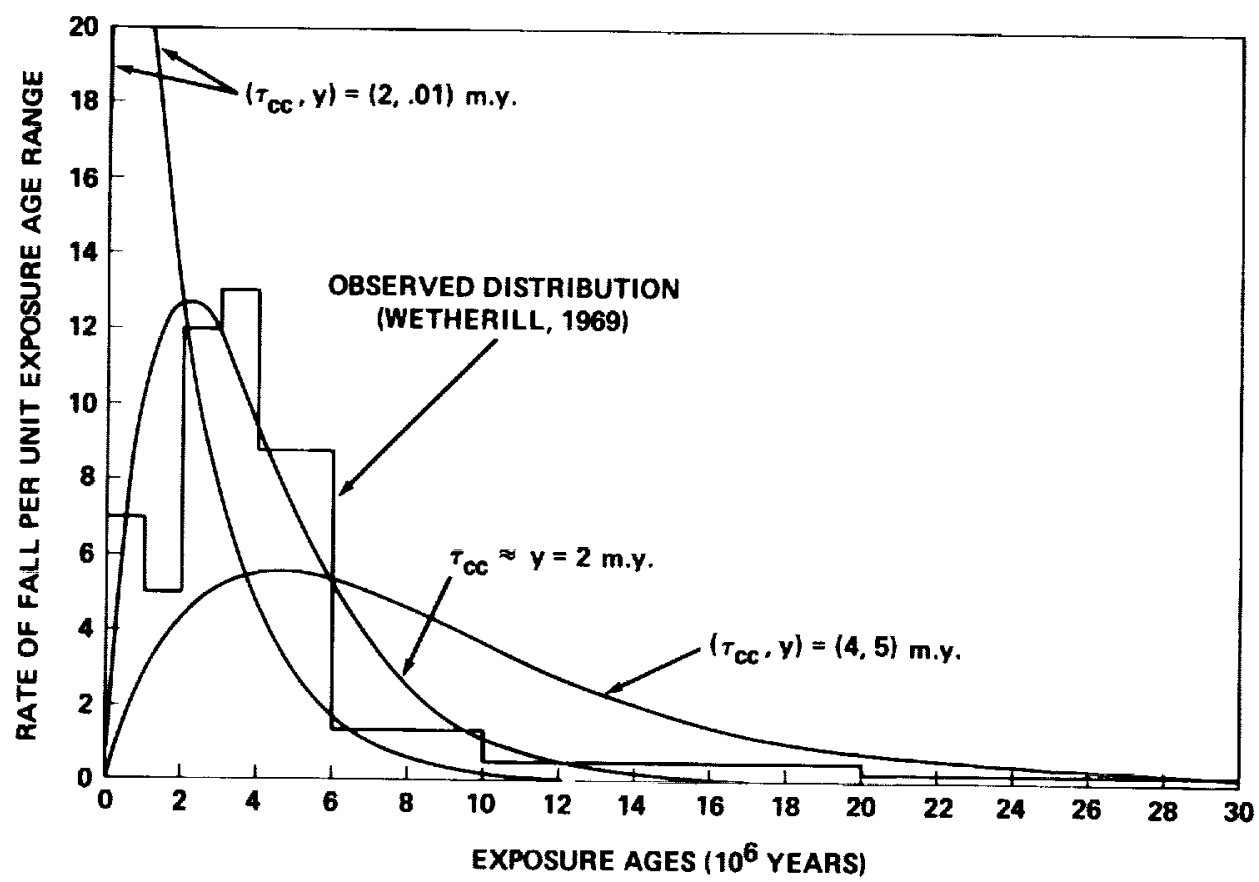

Figvre 6.- Observed and theoretical distribution of cosmic ray exposure ages per unit exposureage range of chondritic meteorites for different survival times $\tau_{c e}$ and $y$ discussed in the text.

Network Project (McCrosky, 1968b), the flux of objects per unit mass range having a mass of 10 tons that enter the Earth's atmosphere is $2.25 \times 10^{-24} \mathrm{~kg}^{-1} \mathrm{~m}^{-2} \mathrm{~s}^{-1}$. Using equation (6) and a mean Farth entry velocity of $20 \mathrm{~km} \mathrm{~s}^{-1}$, we obtain a number density of $2.25 \times 10^{-28}$ particles $/\left(\mathrm{kg} \mathrm{m}^{3}\right)$.

We now assume that the original meteorite parent objects in non-Earth-crossing orbits are objects in Mars-crossing orbits. For the number density per $\mathrm{m}^{3} \mathrm{~kg}$ of Mars-crossing objects having a mass of $10^{4} \mathrm{~kg}$, we employ the results of Kessler (1970), who estimated the number density of large objects at a distance of about $1.5 \mathrm{AU}$ from the Sun to be about 1 percent of the peak number density of the asteroidal belt. Assuming the latter to be about double the average number density (Narin, 1966) and using the results of Dohnanyi (1969), we then get a value of $2.22 \times 10^{-28}$ for the number density $h$ per $\left(\mathrm{kg} \mathrm{m}^{3}\right)$ of $10^{4} \mathrm{~kg}$ objects at a distance of $1.5 \mathrm{AU}$ from the Sun (i.e., Marscrossing orbits). This number is, however, an underestimate, since these Mars-crossing objects spend most of their time away from perihelion and near aphelion. A best estimate for the number density of Mars crossing meteorite parent objects is perhaps an order of magnitude greater.

Using equation (19), we can now calculate the survival time $x$; the result is

$$
\begin{aligned}
x=y\left(h / n_{e}\right) & \approx 10 \times 2\left(2.22 \times 10^{-28} / 2.25 \times 10^{-28}\right) \\
& \approx 2 \times 10^{7} \mathrm{yr}
\end{aligned}
$$

which is surprisingly short. On the other hand, if we used Hawkins' (1963) estimate for the flux of stone meteorite-producing objects, then the number density $n_{c}$ of $10^{4} \mathrm{~kg}$ objects near Earth would be $6.4 \times 10^{-30} /\left(\mathrm{kg} \mathrm{m}^{3}\right)$ and we would have

$$
\begin{aligned}
x & =2 \times 10\left(2.2 \times 10^{-28} / 6.4 \times 10^{-30}\right) 10^{6} \mathrm{yr} \\
& =\approx 7 \times 10^{8} \mathrm{yr}
\end{aligned}
$$

It therefore appears that a diffusion time $x$ ranging from as short a value as $2 \times 10^{7}$ yr up to about $6 \times 10^{9}$ yr can explain the data, depending on the flux law employed, or more specifically, depending on the extent meteorite producing bodies are assumed to contribute to the Prairie Network flux of fireballs.

\section{DISCUSSION AND CONCLUSION}

The considerations of the section on radiation pressure suggest the difficulty of creating micrometcoroids in substantial quantities, either by cometary emission or by the production of second- 
ary ejecta during collisions: Radiation pressure is likely to expel most of the small objects shortly after their creation, while those that initially survive radiation pressure will spiral into the Sun as a result of the Poynting-Robertson effect. While a detailed mathematical formulation of this problem has not been developed, the "bending over" of the flux curve for micrometeoroids (fig. 1) is probably caused by this process.

Meteoroids in the radio and photographic meteor range have survival times limited by catastrophic collisions. Fragments from the disruption of relatively large objects are not produced in sufficient quantities to maintain the population of these metcoroids in steady state (D-I), hence it is necessary that a steady supply of cometary debris be available to replenish the destroyed particles.

Large objects, of the meteorite producing class, have survival times limited by collisions as well as gravitational dispersal. If the latter were unimportant, most meteorites would have a very young radiation exposure age corresponding to the curve in figure 6 , labelled $\left(\tau_{c c}, y\right)=(2, .01)$ m.y.; also, if collisions were very rare and the survival time were dominated by gravitational dispersal alone, a similar exponential type curve would be obtained. The data support neither of these distributions but suggest that the gravitational dispersal time is comparable to the collisional survival time of these objects prior to their having been scatțered into Earth-crossing orbits. This is consistent with a model in which the meteorite parent objects have Mars-crossing orbits and aphelia in the asteroid belt or somewhat beyond (Wetherill, 1968, 1969; Anders, 1971). These objects are then scattered, during close encounters with Mars or perhaps Jupiter, into Earth-crossing orbits. This simple model is found to predict a distribution of meteorite radiation exposure ages consistent with observation (Wetherill, 1969). While a precise identification of these original objects is still under discussion (Wetherill, 1969; Anders, 1971), results derived from this simple statistical model may provide additional clues concerning their identity.

\section{ACKNOWLEDGMENTS}

Thanks are due to M. Liwshitz, R. E. MeCrosky, and G. W. Wetherill for some important suggestions and valuable discussions.

\section{REFERENCES}

ANDErs, E., 1971. Interrelations of meteorites, asteroids and comets, in Physical Studies of Minor Planets, edited by T. Gehrels, NASA SP-267, Supt. of Documents, U.S. Govt. Printing Office, Washington, 429-446.

ArNold, J. R., 1965. The origin of meteorites as small bodies. II. The model. III. General considerations, Asirophys. J., 141, 1536-1556.

Berg, O. E., AND GerlofF, U., 1970. Orbital elements of micrometcoroids derived from Pioneer 8 measurements, J. Geophys. Res., 75, 6932-6939.

Brown, H., 1960. Density and mass distribution of meteorites, $J$. Geophys. Res., 75, 1679-1683.

Dohnany, J. S., 1965. The meteoroid environment of the Apollo program, Bellcomm Repl., TR-66-340-1.

- 1966. Model distribution of photographic meteors, Bellcomm Rept., TR-66-340-1.

_ 1967. Collision model of meteoroids, in The Zodiacal Light and the Interplanetary Medium, edited by J. L. Weinberg, NASA SP-150, Supt. of Documents, U.S. Govt. Printing Office, Washington, 315-319.

- 1969. Collisional model of asteroids and their debris, J. Geophys. Res, 74, 2531-2554; also in Bellcomm Rept. TR-68-710-4, 1968.

- 1970. On the origin and distribution of meteoroids, J. Geophys. Res., 75, 3468-3493.

_, 1971a. Microm teoroids, EOS Trans. A m. Geophys. Union, 52, IUGG, 459-464.

__, 1971b. The lunar micrometeoroid experiment, LO 33, Bellcomm Rept. TM-71-2015-2.

Fechtic, II., Gerloff, U., and Weihrauch, J. H., 1968. Results of cosmic dust collection on Luster 1965, J. Geophys. Res., 73, 5029-5037.

Fleischer, R. L., Hakt, H. R., JR., and Comstock, G. M., 1971. Very heavy solar cosmic rays: energy spectrum and implications for lunar erosion, Science, 171, 1240-1242.

Gault, D. E., Shommaker, E. M., and Moone, J. H., 1963. Spray ejected from the lunar surface by meteoroid impact, NASA Tech. Note D-1767. 
Hartmann, W. K., 1965. Terrestrial and lunar flux of large meteorites in the last two billion years, Icarus, 4, 157-165.

Harwit, M., 1963. Origins of the zodiacal dust cloud, J. Geophys, Res., 68, 2171-2180.

HAwkins, G. S., 1963. Impacts on the earth and moon, Nature, 197, 781.

Jennison, R. C., McDonnflu, J. A. M., And Rodger, I., 1967. The Ariel II micrometeorite penetration measurements, Proc. Roy. Soc. London, A300, 251-269.

KerRidge, J. F., 1970. Micrometeorite environment at the Earth's orbit, Nature, 228, 616-619.

Kessler, D. J., 1970. Meteoroid environment model-170 (interplanetary and planetary), NASA Special Rept. SP-8038.

McCrosky, R. E., 1968a. Orbits of photographic meteors, Physics and Dynamics of Meteors, edited by L. Kresák and P. M. Millman, Springer-Verlag, New York, 265-279.

- 1968 b. Distributions of large meteoric bodies, Smithson. Astrophys. Obs. Spec. Rept. No. $280,1-13$.

McCrosky, R. E., AND Ceplecha, Z., 1970. Fireballs and the physical theory of meteors, Bull. Astron. Inst. Czech., 21, 271-296.

McDonnell, J. A. M., 1971. Review of in situ measurements of cosmic dust particles in space, Space Res., 9, 415-435.

Moore, H. J., ANd Gadut, D. F., 1965. The fragmentation of spheres by projectile impact, Astrogeologic Studies, U.S. Geol. Surv. Ann. Progr. Rept., 127-150.

Moore, H. J., ANd Robertson, F. G., 1966. Hypervelocity impact craters in pumice, Astrogeologic Studies, U.S. Geol. Surv. Ann. Progr. Rept., 107-125.

NARIN, F., 1966. Spatial distribution and motion of the known asteroids, J. Spacecraft Rockets, $3,1438-1440$.

Naumann, R. J., 1968. Calibration of Pegasus and Explorer XXIII detector panels, presented at OART-OSSA Meteoroid Environment Workshop, NASA Headquarters, 342.

Nadmann, R. J., Jex, D. W., and Jounson, C. L., 1969. Calibration of Pegasus and Explorer XXIII detector panels, NASA Tech. Rept. R-321, 1-37.

OPIK, E. J., 1951. Collision probabilitics with the planets and the distribution of interplanetary matter, Proc. Roy. Irish. Acad., 54A, 165-199.

$\longrightarrow, 1958$. On the catastrophic effects of collisions with celestial bodies, Irish Astrophys. $J ., 5$, $34-36$.

— 1966. The stray bodies in the solar system. II. The cometary origin of meteorites, Advan. Astron. Astrophys., Academic Press, New York, 301-336.

Robertson, H. P., 1937. Dynamical effects of radiation on the solar system, Mon. Not. Roy. Astron. Soc., 97, 423-438.

Soberman, R. K., 1971. The terrestrial influx of small meteoric particles, Rev. Geophys. Space Phys., 9, 239-258.

VAN DE Hulst, H. C., 1962. Light Scaltering by Small Particles, J. Wiley and Sons, New York.

Wetherit.., G. W, 1967. Collisions in the asteroid belt, J. Geophys. Res., 72, 2429-2444.

- 1968. Dynamical studies of asteroidal and cometary orbits and their relation to the origin of meteorites, in Origin and Distribution of the Elements, edited by L. H. Ahrens, Pergamon Press, London, 423-443.

- 1969. Relationships between orbits and sources of chondritic metcorites, Meteorite Res., edited by P. M. Millman, Springer-Verlag, New York, 573-589.

Wetherill, G. W., and Wilitams, J. G., 1968. Evaluation of the Apollo asteroids as sources of stone meteorites, $J$. Geophys. Res., 73, 635-648.

Whipple, F. L., 1967. On maintaining the meteoritic complex, in The Zodiacal Light and the Interplanetary Medium, edited by J. L. Weinberg, NASA SP-150, Supt. of Documents, U.S. Govt. Printing Office, Washington, 409-426.

Wyatt, S. P., and Whipple, F. L., 1950. The Poynting-Robertson effect on meteor orbits, Astrophys. J., 111, 134-141. 